\title{
Poverty, depression and resilience as joint predictors of condom use self-efficacy among Northern \& Indigenous youth in Canada
}

Dr. C.H. Logie ${ }^{1 *}$, Dr. C.L. Lys ${ }^{2,3}$, Mr. M. Okumu¹, Ms. J. Fujioka1, Ms. K. Mackay ${ }^{2}$

1: Factor-Inwentash Faculty of Social Work, University of Toronto; 2: Fostering Open eXpression Among Youth (FOXY); 3: Dalla Lana School of Public Health, University of Toronto

Contact: Dr. Carmen Logie, Factor-Inwentash Faculty of Social Work, University of Toronto, Canada. carmen.logie@utoronto.ca

Background: Social and health disparities compromise the wellbeing of Northern and Indigenous adolescents in the Northwest Territories (NWT) where food insecurity, sexually transmitted infections (STI) and depression prevalence are higher than other Canadian regions. Condoms are an accessible STI prevention strategy for adolescents, yet depression and food insecurity may constrain condom use self-efficacy. The potential role of resilience in condom use self-efficacy is understudied among adolescents in Northern Canada.

Methods: This study examined factors associated with condom use self-efficacy among Northern and Indigenous adolescents. We examined: 1) resilience as a mediator in the relationship between depression and condom use self-efficacy; and 2) if food insecurity influenced the indirect effect of depression on condom use self-efficacy through resilience. We conducted a crosssectional tablet based survey in 17 NWT communities with adolescents aged 13-18 who had attended a peer leadership training held by a Northern and Indigenous sexual health agency in the NWT. Participants completed socio-demographic, condom self-efficacy, depressive symptoms, resilience, and food insecurity measures. We conducted descriptive statistics, followed by ordinary least squares regression to test the mediating effect of resilience on the relationship between depression and condom use self-efficacy, and a conditional process analysis to examine the moderating role of food $^{F}$ insecurity in the relationship between depression, resilience, and condom use self-efficacy.

Table 2. Model coefficients for the conditional process model examining food insecurity as a moderator in the relationship between depression, resilience and condom use self-efficacy

among adolescents in the Northwest Territories, Canada $(n=85)$

\begin{tabular}{|c|c|c|c|c|c|c|c|c|}
\hline \multirow[b]{3}{*}{ Predictors } & \multicolumn{8}{|c|}{ Consequent } \\
\hline & & \multicolumn{3}{|c|}{$\begin{array}{c}M \\
\text { (Resilience) }\end{array}$} & & \multicolumn{3}{|c|}{$\begin{array}{c}\mathrm{Y} \\
\text { (Condom use self-efficacy) }\end{array}$} \\
\hline & & coefficient & SE & $p$ & & coefficient & SE & $p$ \\
\hline X (Depression) & a & -1.332 & 0.363 & 0.001 & ć & -0.050 & 0.179 & 0.779 \\
\hline M (Resilience) & & -- & -- & -- & $b$ & -0.111 & 0.070 & 0.113 \\
\hline V (Food insecurity) & & & & & & -11.295 & 4.200 & 0.008 \\
\hline Interaction $(\mathrm{M} \times \mathrm{V})$ & & & & & & 0.245 & 0.098 & 0.014 \\
\hline \multirow[t]{3}{*}{ constant } & $I_{1}$ & 48.943 & 1.763 & $\begin{array}{l}<0.00 \\
0\end{array}$ & i2 & 18.460 & 3.390 & 0.000 \\
\hline & \multicolumn{4}{|c|}{$R^{2}=0.152$} & \multicolumn{4}{|c|}{$\mathrm{R}^{2}=0.325$} \\
\hline & \multicolumn{4}{|c|}{$F=13.467, p=0.001$} & \multicolumn{4}{|c|}{$F=8.681, p=0.000$} \\
\hline
\end{tabular}

Findings: Most participants ( $n=85$; mean age: 15.77, SD: 2.05 ) identified as Indigenous $(n=65 ; 77.4 \%)$ and female $(n=70$; $82.4 \%)$ and reported depressive symptoms $(n=54 ; 70.1 \%)$. The results indicate that resilience mediates the relationship between depression and condom use self-efficacy. There was a significant positive interaction effect between resilience and food insecurity on condom use self-efficacy, suggesting that food insecure participants with higher resilience scores reported higher condom use self-efficacy scores. Findings can inform strengths-based, resilience focused strategies to advance sexual health among Northern adolescents-and underscore the need for poverty reduction strategies.

Table 1. Socio-demographics characteristics of adolescent peer leaders in the Northwest Territories, Canada $(n=85)$

\begin{tabular}{rr} 
Variable & $\mathbf{N}(\%) /$ mean (SD) \\
\hline Age (years) & $15.77(2.05)$
\end{tabular}

Gender (female) $70(82.4 \%)$

Male $10(11.8 \%)$

Other gender

Indigenous identity

$65(77.4 \%)$

Depressive symptoms present $54(70.1 \%)$

Experiencing food insecurity
Figure 1. Examining resilience as a mediator in the relationship between depression and condom use self-efficacy among adolescents in the Northwest Territories, Canada $(\mathrm{n}=85)$ 